\title{
Eccentricity evolution in mean motion resonance and non-radial solar wind
}

\author{
P. Pástor, J. Klačka, J. Petržala, and L. Kómar
}

\begin{abstract}
Department of Astronomy, Physics of the Earth, and Meteorology, Faculty of Mathematics, Physics and Informatics, Comenius University, Mlynská dolina, 84248 Bratislava, Slovak Republic e-mail: pavol.pastor@fmph.uniba.sk
\end{abstract}

Received 4 November 2008 / Accepted 4 March 2009

\section{ABSTRACT}

Eccentricity evolution of a dust particle in a mean motion orbital resonance with a planet in circular orbit is investigated. The action of solar electromagnetic and corpuscular radiation, including non-radial components of the solar wind velocity, is taken into account. Various types of eccentricity evolution depend on the angle between the radial direction and the direction of the solar wind velocity. The evolution changes at the analytically derived angles. Its application to exosolar systems is included.

Key words. Sun: solar wind - interplanetary medium - celestial mechanics - stars: planetary systems

\section{Introduction}

The motion of interplanetary dust particles is influenced by gravitational forces of the Sun and planets and by non-gravitational effects. The effect of solar electromagnetic radiation in the form of the Poynting-Robertson (P-R) effect and the effect of the radial solar wind are conventionally taken into account (see, e.g. Robertson 1937; Wyatt \& Whipple 1950; Whipple 1955; Dohnanyi 1978; Leinert \& Grün 1990; Dermott et al. 2001; Grün 2007).

Physics of resonances with Solar System planets has been investigated mainly for the last three decades. The orbital motion of dust particles in mean motion orbital resonances with a planet has been intensively studied since Jackson \& Zook (1989) predicted a ring of dust particles in resonances with the Earth. The ring was observed by satellites IRAS (Brownlee 1994; Dermott et al. 1994) and COBE (Reach et al. 1995). The orbital evolution of interplanetary dust particles near zones of mean motion resonances was investigated in many papers (Weidenschilling \& Jackson 1993; Beaugé \& Ferraz-Mello 1994; Šidlichovský \& Nesvorný 1994; Liou et al. 1995; Liou \& Zook 1999; Kuchner \& Holman 2003; Klačka \& Pástor 2004). Liou \& Zook (1997) derived the relation between the secular time derivative of eccentricity and the secular time derivative of the inclination of dust particles in mean motion resonance with a planet in a circular restricted three-body problem with the P-R effect and the radial solar wind (the authors incorrectly accounted for the radial solar wind). As for the planar case, it is possible to obtain secular time derivative of a particle's eccentricity using the method presented in Liou \& Zook (1997). The orbital evolution in mean motion resonance in the planar elliptical restricted three-body problem with the P-R effect is discussed in Pástor et al. (2007). The evolution of eccentricity of the particle under the action of the P-R effect and the non-radial solar wind in mean motion resonances with a planet in a circular orbit is investigated in Klačka et al. (2008). Measurement of the non-radial component of the solar wind velocity was performed by Helios 2 during its first solar mission in 1976 (Bruno et al. 2003). According to these results, the angle between the direction of the solar wind velocity and the radial direction is practically independent of heliocentric distance, at least for the distances covered by observations. A constant value of this angle is also in accordance with the results of Parker (1958).

We are motivated mainly by the results of Klačka et al. (2008). We want to obtain detailed predictions of orbital evolution in the orbital resonances when the P-R effect and the nonradial solar wind are considered.

\section{Equation of motion}

Let us consider a spherical dust grain under the action of gravitational forces generated by the Sun and a planet moving around the Sun. Moreover, the grain is evolving under the action of solar electromagnetic radiation and solar wind. The equation of motion of the particle is considered in the form

$$
\begin{aligned}
\frac{\mathrm{d} \boldsymbol{v}}{\mathrm{d} t}= & -\frac{\mu(1-\beta)}{r^{2}} \boldsymbol{e}_{R} \\
& -\beta\left(1+\frac{\eta}{\bar{Q}_{\mathrm{pr}}^{\prime}}\right) \frac{\mu}{r^{2}}\left(\frac{\boldsymbol{v} \cdot \boldsymbol{e}_{R}}{c} \boldsymbol{e}_{R}+\frac{\boldsymbol{v}}{c}\right) \\
& +\beta \frac{\eta}{\bar{Q}_{\mathrm{pr}}^{\prime}} \frac{\mu}{r^{2}}\left\{\left(-\gamma_{T} \frac{\boldsymbol{v} \cdot \boldsymbol{e}_{T}}{c}+\frac{1}{2} \frac{v^{2}}{c u}\right) \boldsymbol{e}_{R}\right. \\
& \left.+\gamma_{T}\left(\frac{u}{c}-\frac{\boldsymbol{v} \cdot \boldsymbol{e}_{R}}{c}\right) \boldsymbol{e}_{T}+\left(\frac{\boldsymbol{v} \cdot \boldsymbol{e}_{R}}{c}\right) \frac{\boldsymbol{v}}{u}\right\} \\
& -\mu \frac{m_{P}}{M_{\odot}}\left\{\frac{\boldsymbol{r}-\boldsymbol{r}_{P}}{\left|\boldsymbol{r}-\boldsymbol{r}_{P}\right|^{3}}+\frac{\boldsymbol{r}_{P}}{\left|\boldsymbol{r}_{P}\right|^{3}}\right\},
\end{aligned}
$$

where $\boldsymbol{v}=v_{R} \boldsymbol{e}_{R}+v_{T} \boldsymbol{e}_{T}$ is velocity vector of the particle, $v=|\boldsymbol{v}|$, $r=|\boldsymbol{r}|, \boldsymbol{e}_{R}=\boldsymbol{r} / r, \boldsymbol{e}_{T}$ is transversal unit vector, $\boldsymbol{r}$ and $\boldsymbol{r}_{P}$ are position vectors of the particle and the planet (mass $m_{P}$ ) with respect to the Sun (mass $M_{\odot}$ ), $\mu=G M_{\odot}, G$ is the gravitational constant, $u$ is the speed of the solar wind $u \approx 400 \mathrm{~km} \mathrm{~s}^{-1}, c$ is the speed of light, $\gamma_{T}=\sin \varepsilon, \varepsilon$ is the angle between the radial direction and the direction of the solar wind velocity (the angle $\varepsilon$ lies 
between 2.3 and 2.9 degrees, according to Bruno et al. (2003), and the value of $\varepsilon$ does not significantly depend on heliocentric distance). Parameter $\beta$ is defined as the ratio of the radial component of the electromagnectic radiation force and the gravitational force between the Sun and the particle with zero velocity

$\beta=\frac{3 L_{\odot} \bar{Q}_{\mathrm{pr}}^{\prime}}{16 \pi c G M_{\odot} R \varrho}=5.763 \times 10^{-4} \frac{\bar{Q}_{\mathrm{pr}}^{\prime}}{R[\mathrm{~m}] \varrho\left[\mathrm{kg} / \mathrm{m}^{3}\right]}$.

$L_{\odot}$ is solar luminosity, $L_{\odot}=3.842 \times 10^{26} \mathrm{~W}$ (Bahcall 2002), $\bar{Q}_{\mathrm{pr}}^{\prime}$ is the efficiency factor for radiation pressure integrated over the solar spectrum and calculated for the radial direction $\left(\bar{Q}_{\mathrm{pr}}^{\prime}=1\right.$ for a perfectly absorbing sphere), $\mathrm{R}$ is the radius of the spherical particle and $\varrho$ its density. $\eta$ is the ratio of solar wind energy to electromagnetic solar energy, both radiated per unit of time

$\eta=\frac{4 \pi r^{2} n_{w} m_{1} u c^{2}}{L_{\odot}}$

where $n_{w}$ is the concentration of the solar wind particles at distance $r$ from the Sun, and $m_{1}$ is the mass of the solar wind particle(s). $\eta \approx 1 / 3$ for the Sun. The force caused by the solar wind is derived using the method presented in Klačka \& Saniga (1993) and Klačka (1994). For details of the P-R effect, we refer to Poynting (1903), Robertson (1937) and Klačka (2008a,b) and Klačka et al. (2009). In the derivation of Eq. (1) it is assumed that $\varepsilon$ is approximately in the interval $\left(-5^{\circ}, 5^{\circ}\right)$. This interval enables us to consider $\cos \varepsilon \approx 1$ in the derivation of Eq. (1). Moreover, we assume that the inclination of a dust particle with respect to the solar equator is approximately in the interval $\left(-5^{\circ}\right.$, $5^{\circ}$ ). More complicated expressions for the non-radial solar wind force should be used outside the above presented intervals.

\section{Mean motion orbital resonances with planets}

A particle is in the $q$ th order exterior mean motion resonance with a planet if the ratio of its mean motion $n$ and the mean motion of the planet $n_{P}$ approximately satisfies the equation $n_{P} / n=(p+q) / p$, where $p$ and $q$ are natural numbers. Similarly, the $q$ th order interior mean motion resonance is defined by the relation $n_{P} / n=p /(p+q)$. The special case mean motion $1 / 1$ resonance corresponds to $q=0$. In terms of orbital periods: $T / T_{P}=(p+q) / p$ for the exterior, $T / T_{P}=p /(p+q)$ for the interior resonance and $T / T_{P}=1$ for mean motion $1 / 1$ resonance.

We can determine the semimajor axis of the particle in the mean motion resonance from Kepler's third law. We have

$a^{3} n^{2}=G M_{\odot}(1-\beta)$,

$a_{P}^{3} n_{P}^{2}=G\left(M_{\odot}+m_{P}\right)$,

where $a, a_{P}$ are semimajor axes of the particle characterized by the optical parameter $\beta$ and a planet with mass $m_{P}$ orbiting the Sun of mass $M_{\odot}$. The first part of Eq. (4) uses the fact that central Keplerian acceleration is given by the sum of solar gravitational acceleration and the dominant radial component of radiation pressure acceleration. With the assumption that $m_{P} \ll M_{\odot}$ we obtain from Eq. (4)

$a=(1-\beta)^{1 / 3}\left(\frac{n_{P}}{n}\right)^{2 / 3} a_{P}$.

On the basis of the definition of mean motion resonance and Eq. (5), we can write

$a=(1-\beta)^{1 / 3}\left(\frac{p+q}{p}\right)^{2 / 3} a_{P}$ for the semimajor axis of the particle in the $q$ th order exterior resonance with a planet of mass $m_{P}$. A similar relation can be obtained for the interior resonance. For mean motion 1/1 orbital resonance we can put $q=0$ in Eq. (6).

\section{Evolution of eccentricity in a mean motion resonance}

A dust particle is captured in a mean motion orbital resonance with a planet and the P-R effect and the non-radial solar wind affect its motion. The secular time derivative of the eccentricity of the particle is (Klačka et al. 2008)

$$
\begin{aligned}
\left\langle\frac{\mathrm{d} e}{\mathrm{~d} t}\right\rangle & =\beta \frac{\mu}{c}\left(1+\frac{\eta}{\bar{Q}_{\mathrm{pr}}^{\prime}}\right) \frac{\left(1-e^{2}\right)^{1 / 2}}{a^{2} e}(X-Y), \\
X & =1-\frac{\left(1+3 e^{2} / 2\right)(1-\beta)^{1 / 2}}{\left(a / a_{P}\right)^{3 / 2}\left(1-e^{2}\right)^{3 / 2}}, \\
Y & =\frac{\gamma_{T}}{\gamma_{C}}\left\{1-\frac{(1-\beta)^{1 / 2}}{\left(a / a_{P}\right)^{3 / 2}\left(1-e^{2}\right)}\right\}, \\
\gamma_{C} & =\frac{1+\eta / \bar{Q}_{\mathrm{pr}}^{\prime}}{\eta / \bar{Q}_{\mathrm{pr}}^{\prime}} \frac{1}{u} \sqrt{\frac{\mu(1-\beta)}{a}},
\end{aligned}
$$

where $\gamma_{T}=\sin \varepsilon$. In the derivation of this equation it is assumed that $m_{P} \ll M_{\odot}$. The ratio $a / a_{P}$ can be expressed through the ratio $n_{P} / n$ from Eq. (5). If the initial eccentricity equals 0 , then the derivation of Eq. (7) does not allow any conclusions since the partial derivative of the Tisserand parameter with respect to eccentricity is 0 (see Klačka et al. 2008).

We define a new function $W(e)$ as

$$
\begin{aligned}
& W(e)=X(e)-Y(e), \\
& X(e)=1-\frac{\left(1+3 e^{2} / 2\right)(1-\beta)^{1 / 2}}{\left(a / a_{P}\right)^{3 / 2}\left(1-e^{2}\right)^{3 / 2}} \\
& Y(e)=\frac{\gamma_{T}}{\gamma_{C}}\left\{1-\frac{(1-\beta)^{1 / 2}}{\left(a / a_{P}\right)^{3 / 2}\left(1-e^{2}\right)}\right\} .
\end{aligned}
$$

The differentiation of the function $W(e)$ with respect to eccentricity is

$\frac{\mathrm{d} W}{\mathrm{~d} e}=\frac{e(1-\beta)^{1 / 2}}{\left(a / a_{P}\right)^{3 / 2}\left(1-e^{2}\right)^{2}}\left\{-\frac{3}{2} \frac{4+e^{2}}{\left(1-e^{2}\right)^{1 / 2}}+2 \frac{\gamma_{T}}{\gamma_{C}}\right\}$

It is easy to show that $\mathrm{d} W / \mathrm{d} e<0$ for all $e \in(0,1)$ and for $\gamma_{T} / \gamma_{C}<3$. Thus, $W(e)$ is a decreasing function for these values. The value $W(e=0)$ is

$W(0)=\left\{1-\left(a_{P} / a\right)^{3 / 2}(1-\beta)^{1 / 2}\right\}\left(1-\gamma_{T} / \gamma_{C}\right)$

\subsection{Exterior mean motion resonances}

Figure 1 depicts the function $W(e)$ for exterior mean motion $4 / 3$ resonance for various values of $\gamma_{T} / \gamma_{C}$. If $W(e)$ is positive, then the eccentricity increases from a given value of eccentricity $e$. If $W(e)$ is negative, then the eccentricity decreases.

For the exterior mean motion resonances, Eq. (10) reduces to

$W(0)=\{1-p /(p+q)\}\left(1-\gamma_{T} / \gamma_{C}\right)$.

The value $W(0)$ is positive or zero for $\gamma_{T} / \gamma_{C} \leq 1$. 


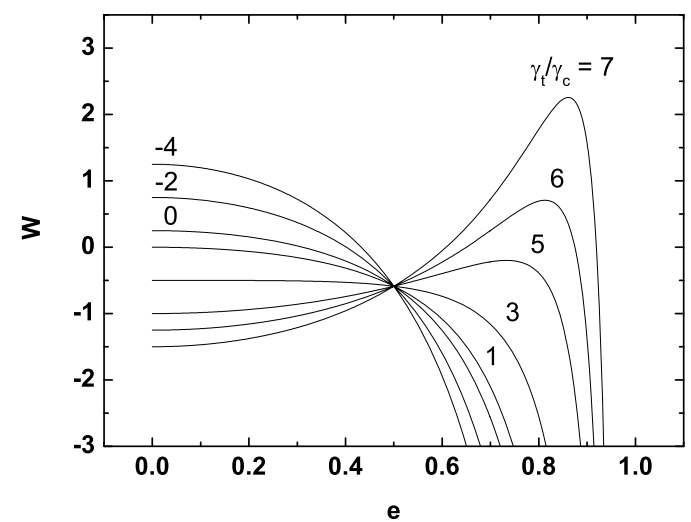

Fig. 1. The function $W(e)$ for exterior mean motion $4 / 3$ resonance. Various values of $\gamma_{T} / \gamma_{C}$ are used.

\subsection{1. $\gamma_{T} / \gamma_{C} \leq 1$}

If $\gamma_{T} / \gamma_{C}<1$, then the function $W(e)$ is a decreasing function of eccentricity (see Eq. (9) and Fig. 1). One root of the function $W(e)$ exists, since $W(0)>0$ and $\lim _{e \rightarrow 1} W(e)=-\infty$. We denote this root as $e_{\mathrm{I}}$. Various functions $W(e)$ in Fig. 1 intersect at eccentricity $e_{y}$ for which is $Y(e)=0 . W\left(e_{y}\right)$ is negative. The statement follows from the inequality

$\frac{\left(1+3 e^{2} / 2\right)(1-\beta)^{1 / 2}}{\left(a / a_{P}\right)^{3 / 2}\left(1-e^{2}\right)^{3 / 2}}>\frac{(1-\beta)^{1 / 2}}{\left(a / a_{P}\right)^{3 / 2}\left(1-e^{2}\right)}$,

which holds for all $e \in[0,1) . e_{y}>e_{\mathrm{I}}$, because $W(e)$ is a decreasing function of eccentricity and $W\left(e_{\mathrm{I}}\right)>W\left(e_{y}\right)$. If $e<e_{\mathrm{I}}$, then $W(e)>0$. Thus, the secular evolution of eccentricity is an increasing function of time and the eccentricity asymptotically increases to the value $e_{\mathrm{I}}$ ( since $\left.\lim _{e \rightarrow e_{\mathrm{I}}}\langle\mathrm{d} e / \mathrm{d} t\rangle=0\right)$. If $e>e_{\mathrm{I}}$, then $W(e)<0$ and the eccentricity asymptotically decreases to the value $e_{\mathrm{I}}$. The value of $e_{\mathrm{I}}$ is a decreasing function of $\gamma_{T} / \gamma_{C}$ (for more details see Appendix A). If $\gamma_{T} / \gamma_{C}$ reaches the value $\gamma_{T} / \gamma_{C}=1$, then $W(0)=0$. Thus, $e_{\mathrm{I}}=0$. We assumed that $-5^{\circ} \lesssim \varepsilon \lesssim 5^{\circ}$, in the derivation of Eq. (1). Thus, for the case $0<\gamma_{C} \lesssim \sin 5^{\circ}$, we can define the angle $\varepsilon_{\text {crit }}$ for which $e_{\mathrm{I}}=0$. We get

$\sin \varepsilon_{\text {crit }}=\gamma_{C}=\frac{1+\eta / \bar{Q}_{\mathrm{pr}}^{\prime}}{\eta / \bar{Q}_{\mathrm{pr}}^{\prime}} \frac{1}{u} \sqrt{\frac{\mu(1-\beta)}{a}}$.

Let us denote $e_{x}$ the value of the eccentricity at which $X(e)=0$ for exterior resonances. We have (see also Liou et al. 1995)

$\frac{p+q}{p}=\frac{1+3 e_{x}^{2} / 2}{\left(1-e_{x}^{2}\right)^{3 / 2}}$.

If the value of $\gamma_{T}$ increases from 0 (the radial solar wind and the P-R effect) to $\sin \varepsilon_{\text {crit }}$, then the value of $e_{\mathrm{I}}$ decreases from $e_{x}$ to 0 (circular orbit).

\subsection{2. $1<\gamma_{T} / \gamma_{C}<3$}

If $1<\gamma_{T} / \gamma_{C}<3$ for an exterior mean motion resonance, then $W(e)$ does not have a root for $e \in[0,1)$, since $W(0)<0$ and $W(e)$ is a decreasing function of eccentricity. The secular evolution of eccentricity is always a decreasing function of time and the eccentricity non-asymptotically decreases to 0 (zero eccentricity can be reached).

\subsection{3. $\gamma_{T} / \gamma_{C} \geq 3$}

If $\gamma_{T} / \gamma_{C} \geq 3$ for exterior mean motion resonances, then $W(0)<$ 0 . The function $W(e)$ also can be an increasing function of eccentricity, but only if the following inequality holds:

$\frac{3}{4} \frac{4+e^{2}}{\left(1-e^{2}\right)^{1 / 2}}<\frac{\gamma_{T}}{\gamma_{C}}$,

see Eq. (9).

Three possibilities exist:

1. $W(e)$ does not have a root for $e \in[0,1)$;

2. $W(e)$ has only one root for $e \in[0,1)$ - we will denote it $e_{\mathrm{II}}$;

3. $W(e)$ has two roots for $e \in[0,1)$ - we will denote them $e_{\mathrm{III}}$ and $e_{\mathrm{IV}}$.

Possibility 1:

The secular evolution of eccentricity is always a decreasing function of time. The eccentricity non-asymptotically decreases to 0 .

Possibility 2:

We can write for $e_{\mathrm{II}}$

$\frac{3}{4} \frac{4+e_{\mathrm{II}}^{2}}{\left(1-e_{\mathrm{II}}^{2}\right)^{1 / 2}}=\frac{\gamma_{T}}{\gamma_{C}}$

since $e_{\mathrm{II}}$ corresponds to the maximum of the function $W(e)$. If the initial eccentricity is greater than $e_{\mathrm{II}}$, then eccentricity asymptotically decreases to the value $e_{\mathrm{II}}$. If the initial eccentricity is less than $e_{\mathrm{II}}$, then the value of the eccentricity non-asymptotically decreases to 0 .

Possibility 3:

In this case we put $e_{\mathrm{III}}<e_{\mathrm{IV}}$. If the initial eccentricity is greater than $e_{\mathrm{IV}}$, then the eccentricity asymptotically decreases to the value $e_{\mathrm{IV}}$. If the initial eccentricity lies between $e_{\mathrm{III}}$ and $e_{\mathrm{IV}}$, the eccentricity asymptotically increases to the value $e_{\mathrm{IV}}$. Finally, if the initial eccentricity is smaller than the value $e_{\mathrm{III}}$, then the eccentricity non-asymptotically decreases to 0 .

\subsection{Interior mean motion resonances}

The graph of the function $W(e)$ for the interior mean motion $2 / 3$ resonance is depicted in Fig. 2. Various values of $\gamma_{T} / \gamma_{C}$ are used.

For interior mean motion resonances, Eq. (10) reduces to

$W(0)=\{1-(p+q) / p\}\left(1-\gamma_{T} / \gamma_{C}\right)$.

The value $W(0)$ is negative for $\gamma_{T} / \gamma_{C}<1$.

$$
\text { 4.2.1. } \gamma_{T} / \gamma_{C}<1
$$

If $\gamma_{T} / \gamma_{C}<1$, then $W(0)<0$ and $W(e)$ is a decreasing function. Secular evolution eccentricity is always a decreasing function of time. The eccentricity non-asymptotically decreases to 0 .

\subsection{2. $\gamma_{T} / \gamma_{C} \geq 1$}

If $\gamma_{T} / \gamma_{C}=1$, then $W(0)=0$. Thus, $e_{\mathrm{V}}=0$ is a root of $W(e)$. The eccentricity asymptotically decreases to $e_{\mathrm{V}}=0$ (circular orbit). For the value $\gamma_{C} \lesssim \sin 5^{\circ}$ we can define (similarly to exterior resonances) an angle $\varepsilon_{\text {crit }}$ for which $e_{\mathrm{V}}=0$. We can calculate $\varepsilon_{\text {crit }}$ for the interior resonances from the condition $\sin \varepsilon_{\text {crit }}=\gamma_{C}$.

If $\gamma_{T} / \gamma_{C}>1$, then $W(0)>0$. In this case, at least one root $e_{\mathrm{V}}$ of $W(e)$ exists. We will show that $W(e)$ has only one root $e_{\mathrm{V}}$ for $e \in[0,1)$. If eccentricity fulfills the inequality given by Eq. (15) 


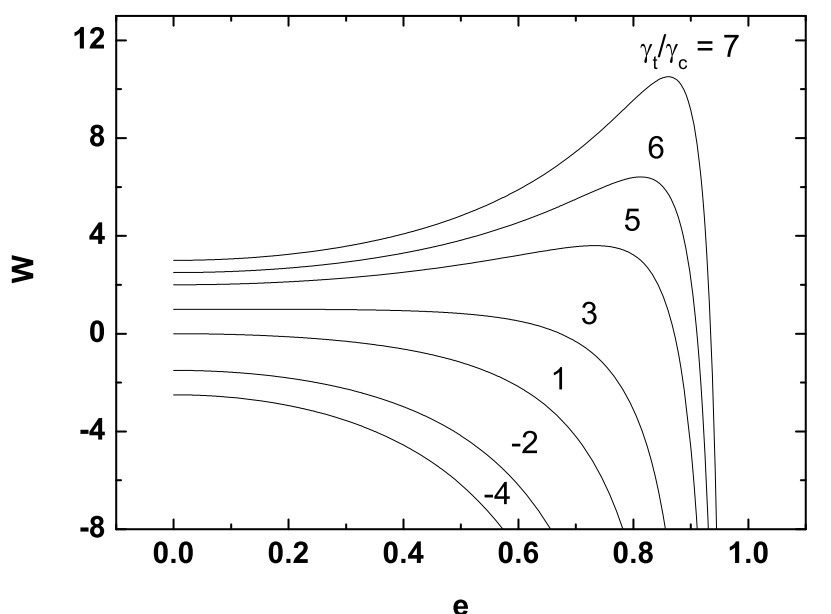

Fig. 2. The function $W(e)$ for interior mean motion $2 / 3$ resonance for various values of $\gamma_{T} / \gamma_{C}$.

(we can apply this inequality also for interior resonances), then $W(e)$ is an increasing function. If the following inequality

$\frac{3}{4} \frac{4+e^{2}}{\left(1-e^{2}\right)^{1 / 2}}>\frac{\gamma_{T}}{\gamma_{C}}$

is fulfilled, then the function $W(e)$ is a decreasing function (see Eq. (9)). Since $W(0)>0$ and $\lim _{e \rightarrow 1} W(e)=-\infty$, the function $W(e)$ has only one root for $e \in[0,1)$. This behavior is also consistent with evolutions for $\gamma_{T} / \gamma_{C}>1$ depicted in Fig. 2 for mean motion $2 / 3$ resonance. $e_{\mathrm{V}}$ is an increasing function of $\gamma_{T} / \gamma_{C}$ (for more details see Appendix B). If the initial eccentricity is greater than the value $e_{\mathrm{V}}$, then the eccentricity asymptotically decreases to the value $e_{\mathrm{V}}$. If the initial eccentricity is less than the value $e_{\mathrm{V}}$, then the eccentricity asymptotically increases to the value $e_{\mathrm{V}}$.

\subsection{Mean motion $1 / 1$ resonance}

Finally, let us consider the special case mean motion $1 / 1$ orbital resonance $(q=0)$. The graph of the function $W(e)$ for $1 / 1$ resonance is shown in Fig. 3. Various values of $\gamma_{T} / \gamma_{C}$ are used.

For the mean motion 1/1 resonance, Eq. (10) reduces to

$W(0)=0$

Thus, one root $e_{\mathrm{VI}}=0$ of the function $W(e)$ always exists, for the mean motion $1 / 1$ resonance.

\subsection{1. $\gamma_{T} / \gamma_{C} \leq 3$}

$W(e)$ is a decreasing function and $W(0)=0$. Thus, $W(e)<0$ for $e \in(0,1)$. The eccentricity asymptotically decreases to 0 .

\subsection{2. $\gamma_{T} / \gamma_{C}>3$}

If $\gamma_{T} / \gamma_{C}>3$, then another root $e_{\mathrm{VII}}$ of $W(e)$ exists, because $W(e)$ is also an increasing function for the values of eccentricity determined by inequality Eq. (15) and $W(0)=0$. If the initial eccentricity is greater than $e_{\mathrm{VII}}$, then the eccentricity asymptotically decreases to $e_{\mathrm{VII}}$. If the initial eccentricity is less than $e_{\mathrm{VII}}$, then the eccentricity asymptotically increases to $e_{\mathrm{VII}}$.

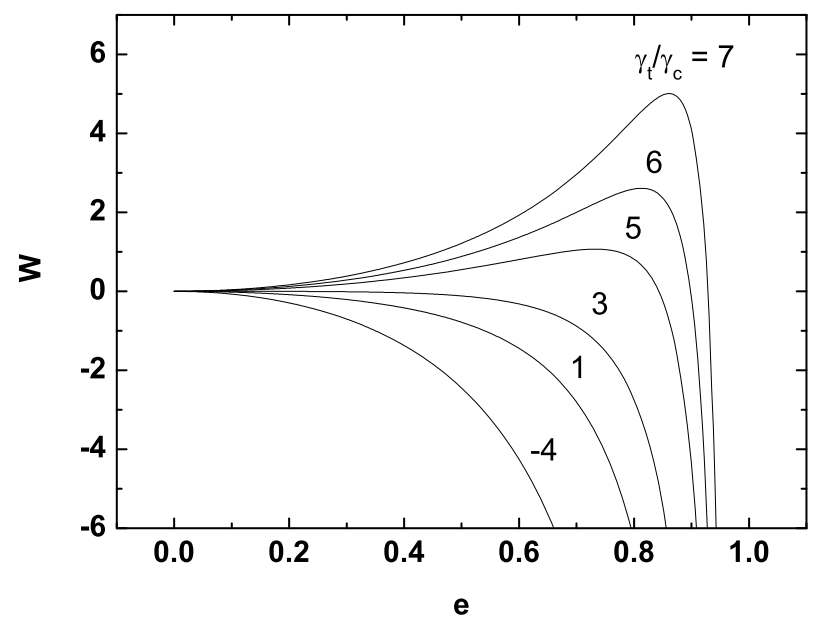

Fig. 3. The function $W(e)$ for mean motion $1 / 1$ resonance for various values of $\gamma_{T} / \gamma_{C}$.

\section{Discussion}

The case $0<\varepsilon<\varepsilon_{\text {crit }}$ yields, qualitatively, the same secular evolution of eccentricity as the case $\varepsilon=0$. For the exterior mean motion resonances, eccentricity exhibits two monotonous parts (an increasing and a decreasing one) asymptotically approaching the limiting value of the eccentricity. For the interior mean motion resonances, eccentricity is a decreasing function of time and the eccentricity non-asymptotically decreases to 0 . The evolution of eccentricity changes for both types of resonances, exterior and interior, at the value $\varepsilon=\varepsilon_{\text {crit }}$. Evolution of the eccentricity changes to a non-asymptotically decreasing evolution for the exterior mean motion resonances, and to an asymptotic behavior for the interior mean motion resonances. $\varepsilon_{\text {crit }}$ represents the smallest value of $\varepsilon$ for which the secular evolution of eccentricity changes (two such values exist for exterior resonances, but only one value for interior resonances). If we use Eq. (6) and a similar equation for the interior resonances, then we get (see Eq. (13))

$\sin \varepsilon_{\text {crit }}=\frac{1+\eta / \bar{Q}_{\mathrm{pr}}^{\prime}}{\eta / \bar{Q}_{\mathrm{pr}}^{\prime}} \frac{\sqrt{\mu / a_{P}}}{u}(1-\beta)^{1 / 3}\left(\frac{p}{p+q}\right)^{1 / 3}$,

for the exterior resonances and

$\sin \varepsilon_{\mathrm{crit}}=\frac{1+\eta / \bar{Q}_{\mathrm{pr}}^{\prime}}{\eta / \bar{Q}_{\mathrm{pr}}^{\prime}} \frac{\sqrt{\mu / a_{P}}}{u}(1-\beta)^{1 / 3}\left(\frac{p+q}{p}\right)^{1 / 3}$,

for the interior resonances.

Table 1 presents the values of $\varepsilon_{\text {crit }}$ for $\beta=0.01, \eta=1 / 3$, $u=400 \mathrm{~km} \mathrm{~s}^{-1}, \bar{Q}_{\mathrm{pr}}^{\prime}=1 / 2,1$ and $3 / 2$, for several exterior and interior resonances with a planet in a circular orbit of radius $a_{P}=30.058 \mathrm{AU}$ (approximately the semimajor axis of planet Neptune).

Figures 4 and 5 depict secular evolution of the particle's eccentricity in the exterior mean motion $4 / 3$ resonance with a planet in a circular orbit of radius $a_{P}=30.058 \mathrm{AU}$ for the case $\beta=0.01$ and $\eta / \bar{Q}_{\mathrm{pr}}^{\prime}=2 / 3$. Figure 4 holds for $\varepsilon=0^{\circ}$ and Fig. 5 holds for $\varepsilon=2.5^{\circ}$. If $0 \leq \varepsilon<\varepsilon_{\text {crit }}=1.762^{\circ}$, then the evolution of the eccentricity is similar to Fig. 4. If $1.762^{\circ}<\varepsilon<5.293^{\circ}$, then the secular evolution of the eccentricity is similar to Fig. 5. The detailed numerical integrations of Eq. (1) are consistent with Eq. (7). Evolutions depicted in Figs. 4 and 5 are calculated from the numerical solution of Eq. (7). 
Table 1. Values of $\varepsilon_{\text {crit }}$ (in degrees) for various exterior and interior resonances with a planet in a circular orbit of radius $a_{P}=30.058 \mathrm{AU}$. The particle is characterized by $\beta=0.01$ and the solar wind by $\eta=1 / 3$ and $u=400 \mathrm{~km} \mathrm{~s}^{-1}$.

\begin{tabular}{cccc}
\hline \hline $\begin{array}{c}\text { Resonant } \\
\text { type }\end{array}$ & $\begin{array}{c}\varepsilon_{\text {crit }} \\
\bar{Q}_{\mathrm{pr}}^{\prime}=1 / 2\end{array}$ & $\begin{array}{c}\varepsilon_{\text {crit }} \\
\bar{Q}_{\mathrm{pr}}^{\prime}=1\end{array}$ & $\begin{array}{c}\varepsilon_{\text {crit }} \\
\bar{Q}_{\mathrm{pr}}^{\prime}=3 / 2\end{array}$ \\
\hline $2 / 1$ & 1.539 & 2.463 & 3.388 \\
$3 / 2$ & 1.694 & 2.711 & 3.729 \\
$4 / 3$ & 1.762 & 2.820 & 3.879 \\
$5 / 4$ & 1.800 & 2.881 & 3.963 \\
$6 / 5$ & 1.825 & 2.921 & 4.018 \\
$1 / 2$ & 2.444 & 3.912 & 5.383 \\
$2 / 3$ & 2.220 & 3.554 & 4.889 \\
$3 / 4$ & 2.135 & 3.417 & 4.700 \\
$4 / 5$ & 2.089 & 3.344 & 4.600 \\
$5 / 6$ & 2.061 & 3.299 & 4.538 \\
\hline
\end{tabular}

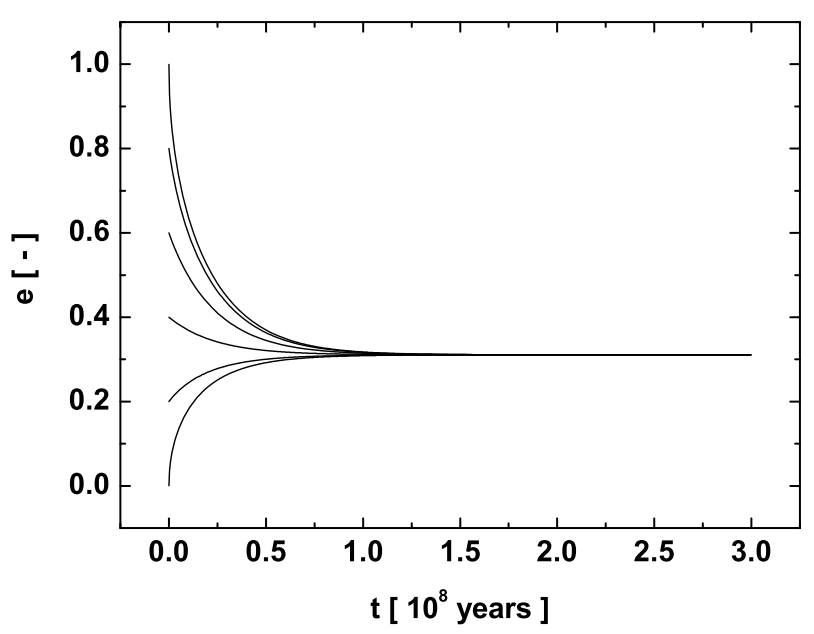

Fig. 4. Evolution of eccentricity obtained from Eq. (7). A dust particle with $\beta=0.01$ and $\eta / \bar{Q}_{\mathrm{pr}}^{\prime}=2 / 3$ is captured in the exterior mean motion $4 / 3$ orbital resonance with a planet of semimajor axis $a_{P}=30.058 \mathrm{AU}$ and eccentricity $e_{P}=0$. Solar wind parameter $\varepsilon=0$. Evolution curve for various initial conditions of eccentricity are shown.

Figure 6 depicts the evolution of eccentricity for the case when the function $W(e)$ has two roots $\left(\gamma_{T} / \gamma_{C}>3\right)$. Various evolutions differ only in initial values of eccentricity. A dust particle with $\beta=0.01$ and $\eta / \bar{Q}_{\mathrm{pr}}^{\prime}=5 / 3$ is captured in the exterior mean motion $5 / 4$ resonance with an artificial planet in a circular orbit of radius $a_{P}=80 \mathrm{AU}$. The speed of the solar wind is $u=400 \mathrm{~km} \mathrm{~s}^{-1}$ and the angle between the radial direction and the direction of the solar wind velocity is $\varepsilon=4^{\circ}$. An asymptotic approach to the limiting value $e_{\mathrm{IV}} \approx 0.8613$ is evident. The evolution of eccentricity is always a decreasing function of time, below the initial eccentricity $e_{\mathrm{III}} \approx 0.5974$.

Figure 7 depicts results of numerical integration of Eq. (1). A particle with $\beta=0.01$ and $\bar{Q}_{\mathrm{pr}}^{\prime}=1 / 5$ is captured in a mean motion $5 / 4$ resonance with a planet of mass $m_{P}=20 m_{\text {Earth }}$ $\left(m_{\text {Earth }} \approx 5.9742 \times 10^{24} \mathrm{~kg}\right)$ in a circular orbit with a semimajor axis $80 \mathrm{AU}$. The solar wind is characterized by $u=400 \mathrm{~km} \mathrm{~s}^{-1}$, $\eta=1 / 3, \varepsilon=4^{\circ}$. As the mass of the star we use solar mass. Initial conditions of the particle are $a=a_{5 / 4} 0.01-5 \times 10^{-5} \mathrm{AU}$, $e=0.6, \omega=0, f=309.6^{\circ}$, where $a_{5 / 4} 0.01$ is the semimajor axis calculated from Eq. (6) using $p=4, q=1$ and $\beta=0.01$. $\omega$ is the argument of perihelion and $f$ is the true anomaly. The planet's initial position is on the $\mathrm{X}$-axis (the axis from which the argument of perihelion is measured). The initial eccentricity of the

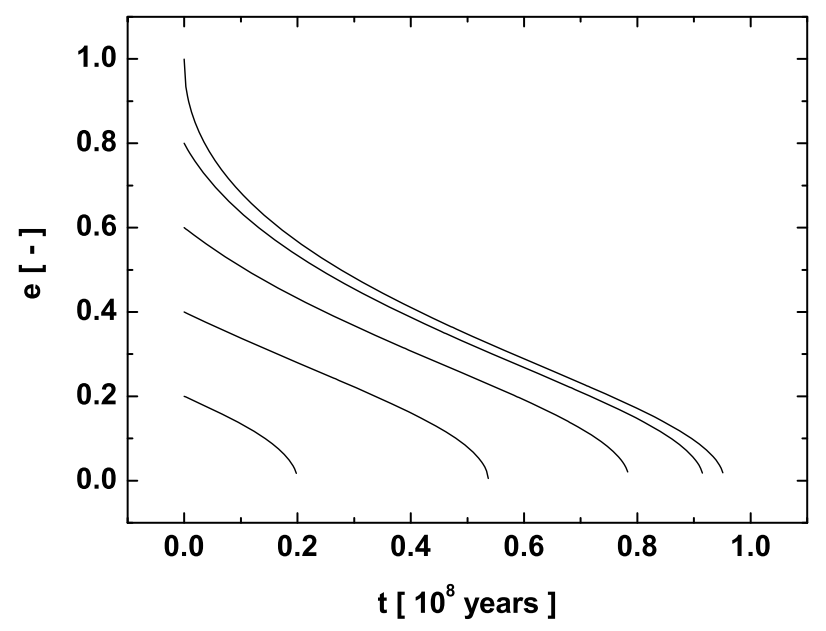

Fig. 5. Evolution of eccentricity obtained from Eq. (7). A dust particle with $\beta=0.01$ and $\eta / \bar{Q}_{\mathrm{pr}}^{\prime}=2 / 3$ is captured in the exterior mean motion $4 / 3$ orbital resonance with a planet of semimajor axis $a_{P}=30.058 \mathrm{AU}$ and eccentricity $e_{P}=0$. The solar wind parameter $\varepsilon=2.5^{\circ}$.

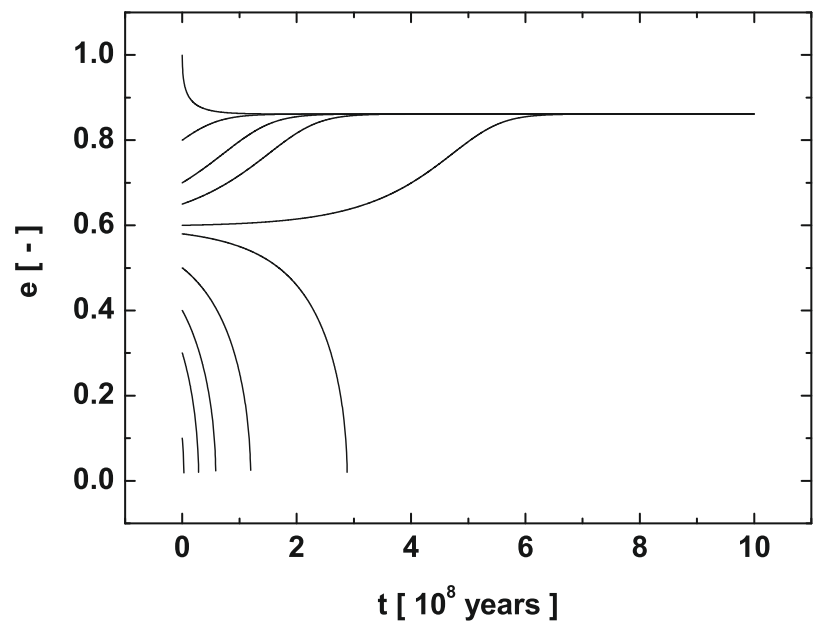

Fig. 6. Evolution of eccentricity of a dust particle with $\beta=0.01$ and $\eta / \bar{Q}_{\mathrm{pr}}^{\prime}=5 / 3$ captured in the exterior mean motion $5 / 4$ orbital resonance with a planet of semimajor axis $a_{P}=80 \mathrm{AU}$ and eccentricity $e_{P}=$ 0 . Solar wind parameter $\varepsilon=4^{\circ}$. Evolution curves for various initial eccentricity are shown.

fifth evolution of eccentricity from the top of Fig. 6 is also 0.6. This evolution of eccentricity corresponds to the evolution of eccentricity obtained by numerical integration of Eq. (1) shown in Fig. 7. Numerical integration shown in Fig. 7 is in accordance with our analytical theory, since evolution of eccentricity asymptotically increases to the value $e_{\mathrm{IV}} \approx 0.8613$.

We found that the function $W(e)$ can have two roots for the first order exterior resonances also for smaller values of $\varepsilon$ if we use greater $p$ (when the other parameters in the function $W(e)$ are constant). But the resonance overlap begins to be significant for the first order exterior resonances with higher $p$ (see e.g. Murray $\&$ Dermott 1999). The particle is captured into a $1 / 1$ resonance with a higher probability than into the first order exterior resonance with a high value of $p$.

Now, we find how $\gamma_{C}$ depends on the individual physical quantities characterizing the star, planetary orbit and dust grain. 

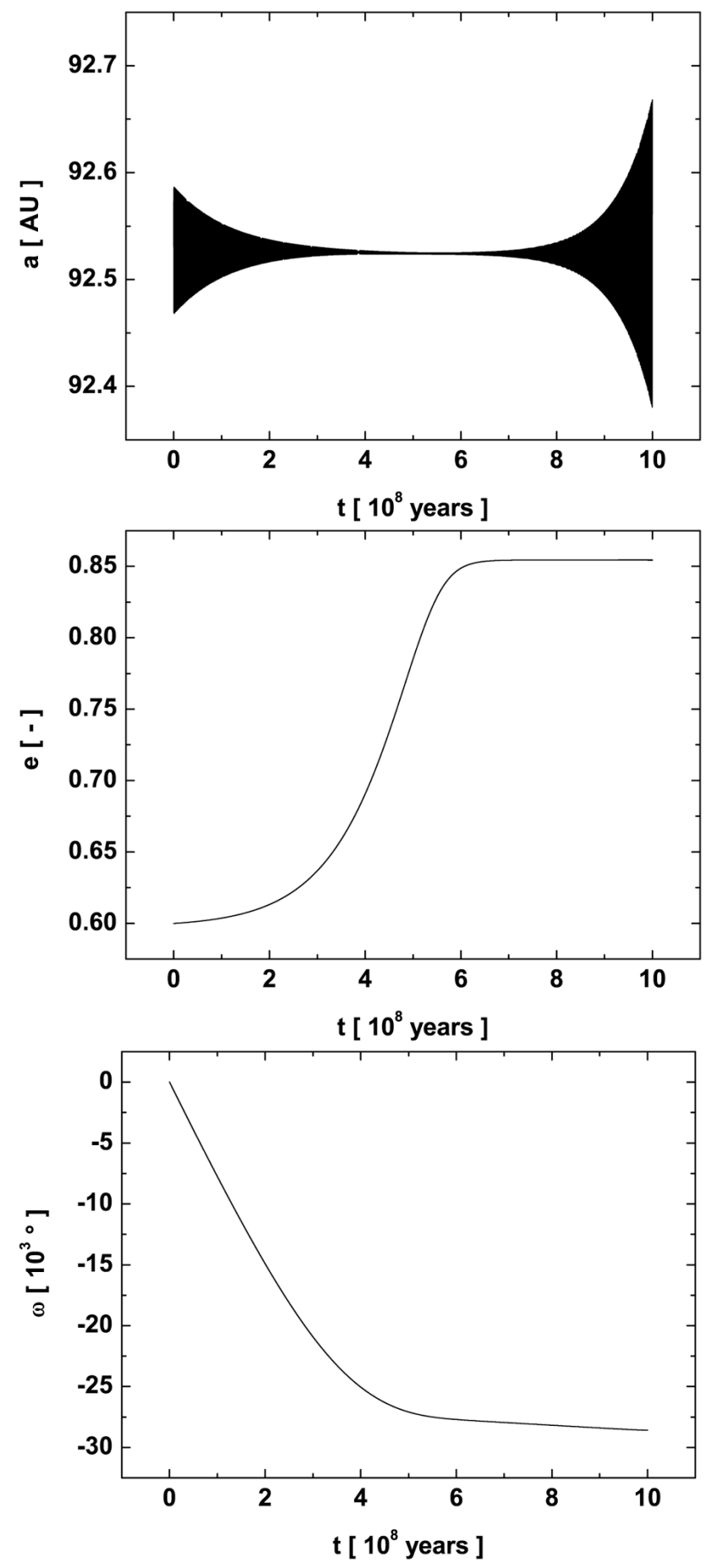

Fig. 7. Orbital evolution of a spherical dust particle with $\beta=0.01$ and $\eta / \bar{Q}_{\mathrm{pr}}^{\prime}=5 / 3$ captured in the exterior mean motion $5 / 4$ resonance with a planet of mass $m_{P}=20 m_{\text {Earth }}$ in a circular orbit with a semimajor axis $a_{P}=80 \mathrm{AU}$. Solar wind parameter $\varepsilon=4^{\circ}$.

We will carry out our analysis for a given resonance, defined by numbers $p$ and $q$. Putting Eqs. (2) and (3) into Eq. (7) yields

$$
\begin{aligned}
\gamma_{C}= & \frac{1+n_{l} m_{1} u c^{2} /\left(L \bar{Q}_{\mathrm{pr}}^{\prime}\right)}{n_{l} m_{1} u c^{2} /\left(L \bar{Q}_{\mathrm{pr}}^{\prime}\right)} \frac{1}{u} \sqrt{\frac{G M}{a_{P}}}\left(\frac{n}{n_{P}}\right)^{1 / 3} \\
& \times\left(1-\frac{3 L \bar{Q}_{\mathrm{pr}}^{\prime}}{16 \pi c G M R_{\varrho}}\right)^{1 / 3}
\end{aligned}
$$

using Eq. (5) and the notation $4 \pi r^{2} n_{w}=n_{l}$. We may assume that the value of $n_{l}$ is a constant independent of the distance from the star. Equation (22) also provides a dependence of $\sin \varepsilon_{\text {crit }}=\gamma_{C}$ on physical quantities characterizing a given system.

Now, we can find how $\gamma_{C}$ depends on individual variables. It is evident that the value of $\gamma_{C}$ decreases with an increasing value of the semimajor axis of the planet $a_{P}$ (see Eq. (22)). $\gamma_{C}$ is an increasing function of a particle's radius $R$ and mass density $\varrho$. The function $f_{1}\left(x_{1}\right)=\left(1+a_{1} x_{1}\right) /\left(a_{1} x_{1}\right)$ has a derivative with respect to $x_{1}$ equal to $f_{1}^{\prime}\left(x_{1}\right)=-1 /\left(a_{1} x_{1}^{2}\right) . f_{1}\left(x_{1}\right)$ is a decreasing function for positive values of $a_{1}$. Thus, $\gamma_{C}$ will be a decreasing function of $n_{l}$ and $m_{1}$. The function $f_{2}\left(x_{2}\right)=\sqrt{x_{2}}\left(1-a_{2} / x_{2}\right)^{1 / 3}$ has a derivative equal to $f_{2}^{\prime}\left(x_{2}\right)=\left(3-a_{2} / x_{2}\right) /\left[6 \sqrt{x_{2}}\left(1-a_{2} / x_{2}\right)^{2 / 3}\right]$. $a_{2} / x_{2}=\beta$. Since $\beta<1$ for particles captured in a mean motion resonance, we obtain $3-\beta>0$. Thus, $\gamma_{C}$ will be an increasing function of $M$. The function $f_{3}\left(x_{3}\right)=\left(1+a_{3} x_{3}\right) /\left(a_{3} x_{3}^{2}\right)$ has a derivative equal to $f_{3}^{\prime}\left(x_{3}\right)=-\left(2+a_{3} x_{3}\right) /\left(a_{3} x_{4}^{3}\right) . f_{3}$ is a decreasing function of $x_{3}$, for positive $a_{3}$ and $x_{3}$. Thus, $\gamma_{C}$ is a decreasing function of $u$. The function $f_{4}\left(x_{4}\right)=\left(1+a_{4} / x_{4}\right)(1-$ $\left.b_{4} x_{4}\right)^{1 / 3} /\left(a_{4} / x_{4}\right)$ has a derivative equal to $f_{2}^{\prime}\left(x_{4}\right)=\left(3-a_{4} b_{4}-\right.$ $\left.4 b_{4} x_{4}\right) /\left[3 a_{4}\left(1-b_{4} x_{4}\right)^{2 / 3}\right], a_{4}>0$ and

$3-a_{4} b_{4}-4 b_{4} x_{4}=3-\frac{\beta \eta}{\bar{Q}_{\mathrm{pr}}^{\prime}}-4 \beta$.

Thus, $\gamma_{C}$ can be an increasing or a decreasing function of $L$ and $\bar{Q}_{\mathrm{pr}}^{\prime}$ for arbitrary values of $\beta, \eta$ and $\bar{Q}_{\mathrm{pr}}^{\prime}$.

We can conclude that $\gamma_{C}$ will be always a decreasing function of $a_{P}, n_{l}, m_{1}, u$, and, an increasing function of $M, R, \varrho$. Equation (22) yields that $\gamma_{C}$ may be neither an increasing nor a decreasing function of $L$ and $\bar{Q}_{\mathrm{pr}}^{\prime}$ if the other parameters are fixed (we do not take into account that luminosity of a star may depend on its mass).

To obtain the evolution presented in Fig. 6 we need to minimize the value of $\gamma_{C}$ or maximize value of $\gamma_{T}$ in order to have the maximum of the value $\gamma_{T} / \gamma_{C}$. Thus, $\gamma_{T} / \gamma_{C}$ is larger for stars with strong stellar wind (high $n_{l}, m_{1}, u$ ) or with large a value of $\varepsilon$. The value of $\gamma_{T} / \gamma_{C}$ can be sufficiently high also for a particle in a resonance with a planet in orbit with a large semimajor axis $a_{P}$. Known systems with a star similar to the Sun and a planet in an orbit with large a semimajor axis are: GQ Lup $(M=$ $0.8 \pm 0.2 M_{\odot}$, Seperuelo et al. 2008; $a_{P} \approx 100 \mathrm{AU}$, Neuhäuser et al. 2005), 1RXS J160929.1-210524 ( $M=0.85_{-0.10}^{+0.20} M_{\odot}$; $a_{P} \approx 330 \mathrm{AU}$, Lafreniere et al. 2008). Also AB Pic has a planet candidate at a separation of $\approx 258 \mathrm{AU}$ (Chauvin et al. 2005). But AB Pic is classified as a dwarf star, similar to many known systems with large semimajor axes. If we calculate $\gamma_{T} / \gamma_{C}$ for an exosolar system and its value is higher than 3 , then secular evolution of the particle's eccentricity in an exterior mean motion resonance can be similar to some of the evolutions shown in Fig. 6.

\section{Conclusion}

We investigated the evolution of eccentricity of an interplanetary spherical dust particle under the action of resonant perturbation of a planet in a circular orbit, the P-R effect and non-radial solar wind.

Eccentricity asymptotically approaches (increases or decreases) a limiting value in exterior mean motion resonances (simple asymptotic behavior) if the P-R effect and radial solar wind are considered. If also the non-radial component of the solar wind velocity is taken into account, then various possibilities 
exist. If the angle between the radial direction and the velocity of the solar wind fulfills $0<\gamma_{T} / \gamma_{C}<1$, then the eccentricity evolution is similar to the case of a radial solar wind. If $1 \leq \gamma_{T} / \gamma_{C}<3$, then the eccentricity is a decreasing function of time and the eccentricity non-asymptotically decreases to zero.

For interior mean motion orbital resonances, eccentricity is always a non-asymptotically decreasing function of time for the $\mathrm{P}-\mathrm{R}$ effect and the radial solar wind. The behavior of particle's eccentricity is inverse to the behavior in the exterior resonances for $0 \leq \gamma_{T} / \gamma_{C}<3$. If we take into account the non-radial component of the solar wind velocity and $0<\gamma_{T} / \gamma_{C}<1$, then the evolution of eccentricity is similar to the case of the radial solar wind. If $1 \leq \gamma_{T} / \gamma_{C}<3$, then eccentricity asymptotically approaches the limiting value (simple asymptotic behavior).

If $\gamma_{T} / \gamma_{C} \geq 3$, then the eccentricity non-asymptotically decreases to 0 or may asymptotically decrease or increase to a limiting value, for exterior resonances. If $\gamma_{T} / \gamma_{C} \geq 3$, then the eccentricity evolution has a simple asymptotic behavior for interior resonances.

For the special case of the mean motion $1 / 1$ resonance, the eccentricity asymptotically decreases to 0 , for $\gamma_{T} / \gamma_{C} \leq 3$. If $\gamma_{T} / \gamma_{C}>3$, then simple asymptotic behavior of the eccentricity exists.

For the case $\gamma_{T} / \gamma_{C}<0$, the evolution of eccentricity of a particle captured in an exterior mean motion resonance is characterized by simple asymptotic behavior. Eccentricity nonasymptotically decreases to 0 in an interior resonance. The special case of the mean motion $1 / 1$ resonance is characterized by an asymptotic decrease of eccentricity to 0 .

We have not found eccentricity evolution characterized by two roots of the function $W(e)$, for a dust particle under the action of electromagnetic and corpuscular radiation of the Sun in an exterior mean motion resonance with a planet in the Solar System.

The values of $\gamma_{C}, \gamma_{T}$ and numbers $p$ and $q$ determine types of eccentricity evolution. The value of $\gamma_{C}$ is:

i) an increasing function of mass of a star and particle's radius and mass density,

ii) a decreasing function of stellar wind speed, mass and concentration of the stellar wind particles, and

iii) an increasing or a decreasing function of luminosity of the star and particle's dimensionless efficiency factor of radiation pressure.

We suggest that eccentricity evolution characterized by two roots of the function $W(e)$ for exterior resonances (eccentricity nonasymptotically decreases to 0 or asymptotically decreases or increases to a limiting value) can be present in an exosolar system with planet(s). This kind of eccentricity evolution is reasonably expected in systems with a strong non-radial stellar wind or in systems with modal non-radial stellar wind with a planet in an orbit characterized by a large semi-major axis and low eccentricity.

Acknowledgements. The authors would like to thank the anonymous reviewer for fruitful comments. The paper was supported by the Scientific Grant Agency VEGA grant No. 2/0016/09 and by the Comenius University grants $\mathrm{UK} / 405 / 2009$.

\section{Appendix A: Exterior mean motion resonances $-e_{1}$}

We will show how the value of the root $e_{\mathrm{I}}$ depends on $\gamma_{T} / \gamma_{C}$. We calculate derivatives of the functions $X(e)$ and $Y(e)$ with respect to the eccentricity

$$
\begin{aligned}
& \frac{\mathrm{d} X}{\mathrm{~d} e}=-\frac{3 e(1-\beta)^{1 / 2}\left(4+e^{2}\right)}{2\left(a / a_{P}\right)^{3 / 2}\left(1-e^{2}\right)^{5 / 2}}, \\
& \frac{\mathrm{d} Y}{\mathrm{~d} e}=-\frac{\gamma_{T}}{\gamma_{C}} \frac{2 e(1-\beta)^{1 / 2}}{\left(a / a_{P}\right)^{3 / 2}\left(1-e^{2}\right)^{2}} .
\end{aligned}
$$

\section{A.1. $\gamma_{T} / \gamma_{C}<0$}

According to Eq. (9), $W(e)$ is a decreasing function of eccentricity, for $\gamma_{T}<0$. Derivatives of the functions $X(e)$ and $-Y(e)$ fulfill $\mathrm{d} X / \mathrm{d} e<0$ and $-\mathrm{d} Y / \mathrm{d} e<0$. Thus, the functions $X(e)$ and $-Y(e)$ are decreasing functions of eccentricity.

Inserting $e_{x}$ (given by Eq. (14)) into the function $W(e)$, we obtain $W\left(e_{x}\right)>0$. The statement follows from the inequality Eq. (12). This means that $e_{\mathrm{I}}$ must be greater than $e_{x}$, since the function $W(e)$ is a decreasing function of eccentricity. If we assume that eccentricity is greater than $e_{x}$ and less than $e_{\mathrm{I}}$, then $X(e)<0$ and $W(e)=X(e)-Y(e)>0$, thus $-Y(e)>0$. Both $X(e)$ and $-Y(e)$ decrease with increasing eccentricity. A positive value of $-Y(e)$ is proportional to $\gamma_{T} / \gamma_{C}$. If we decrease the ratio $\gamma_{T} / \gamma_{C}$, then the value of $X(e)<0$ must decrease more rapidly in order to reach the condition $X(e)-Y(e) \approx 0$. If $X(e)$ and $Y(e)$ approach such values that $X(e) \approx Y(e)$, then the eccentricity $e$ approaches $e_{\mathrm{I}}$. The condition will be fulfilled since $\lim _{e \rightarrow 1} X(e)=-\infty$ and $\lim _{e \rightarrow 1}-Y(e)=-\infty$ (only decreasing $X(e)$ and $-Y(e)$ is not sufficient to fulfill this condition). Thus, $e_{\mathrm{I}}$ will be a decreasing function of $\gamma_{T} / \gamma_{C}$.

\section{A.2. $0 \leq \gamma_{T} / \gamma_{C} \leq 1$}

Derivatives of functions $X(e)$ and $Y(e)$ with respect to eccentricity are $\mathrm{d} X / \mathrm{d} e<0$ and $\mathrm{d} Y / \mathrm{d} e \leq 0$ (see Eqs. (A.1) and (A.2)). Thus, the functions $X(e)$ and $Y(e)$ are decreasing functions of eccentricity. It is easy to show that $\mathrm{d} X / \mathrm{d} e<\mathrm{d} Y / \mathrm{d} e$ for all $e \in$ $(0,1)$, if $0 \leq \gamma_{T} / \gamma_{C}<3$.

Inserting $e_{x}$ into the function $W(e)$, for the case $0<\gamma_{T} / \gamma_{C}<$ 1 , we obtain $W\left(e_{x}\right)<0$. This means that $e_{\mathrm{I}}$ must be smaller than $e_{x}$, since the function $W(e)$ is a decreasing function of eccentricity. If we assume that eccentricity is greater than $e_{\mathrm{I}}$ and less than $e_{x}$, then $X(e)>0$ and $Y(e)>0$. We have also $W(e)=X(e)-Y(e)<0$, i.e. $X(e)<Y(e) . X(e)$ increases faster than $Y(e)$ for decreasing $e$. If $X(e)$ and $Y(e)$ approach such values that $X(e) \approx Y(e)$, then the eccentricity $e$ approaches $e_{\mathrm{I}}$. The value of $Y(e)$ is proportional to $\gamma_{T} / \gamma_{C}$. The greater $\gamma_{T} / \gamma_{C}$ the more rapid an increase of $X(e)$ is necessary in order to reach the condition $X(e)-Y(e) \approx 0$. Thus, $e_{\mathrm{I}}$ is a decreasing function of $\gamma_{T} / \gamma_{C}$. If the value of $\gamma_{T}$ increases from 0 to $\gamma_{C}$, then the value of $e_{\mathrm{I}}$ decreases from $e_{x}$ to 0 .

\section{Appendix B: Interior mean motion resonances $-e_{\mathrm{V}}$}

The root $e_{\mathrm{V}}$ was derived for $\gamma_{T} / \gamma_{C} \geq 1$. Derivatives of the functions $X(e)$ and $Y(e)$ are $\mathrm{d} X / \mathrm{d} e<0$ and $\mathrm{d} Y / \mathrm{d} e<0$ (see Eqs. (A.1) and (A.2)). Thus, the functions $X(e)$ and $Y(e)$ are decreasing functions of eccentricity.

If $\gamma_{T} / \gamma_{C} \geq 1$, inequalities $X(e)<0$ and $Y(e)<0$ hold for the case of the interior resonances. If the initial eccentricity is smaller than $e_{\mathrm{V}}$, then $W(e)>0$, and thus $-X(e)<-Y(e)$. If the 
inequality represented by Eq. (18) is fulfilled, then the function $-X(e)$ increases faster than the function $-Y(e)$, with an increasing eccentricity (see Eqs. (A.1) and (A.2)). This is necessary in order to reach the condition $X(e)-Y(e) \approx 0$. The value of $-Y(e)$ is proportional to $\gamma_{T} / \gamma_{C}$, and, thus, $e_{\mathrm{V}}$ is an increasing function of $\gamma_{T} / \gamma_{C}$.

\section{References}

Bahcall, J. N. 2002, Phys. Rev. C, 65, 025801

Beaugé, C., \& Ferraz-Mello, S. 1994, Icarus, 110, 239

Brownlee, D. E. 1994, Nature, 369, 706

Bruno, R., Carbone, V., Sorriso-Valvo, L., \& Bavassano, B. 2003, J. Geophys. Res., 108(A3), 1130

Chauvin, G., Lagrange, A.-M., Zuckerman, B., et al. 2005, A\&A, 438, L29

Dermott, S. F., Jayaraman, S., Xu, Y. L., Gustafson, B. A. S., \& Liou, J. C. 1994, Nature, 369, 719

Dermott, S. F., Grogan, K., Durda, D. D., et al. 2001, in Interplanetary Dust, ed. E. Grün, B. A. S. Gustafson, S. F. Dermott, \& H. Fechtig (Berlin: SpringerVerlag), 569

Dohnanyi, J. S. 1978, Particle dynamics, in Cosmic Dust, ed. J. A. M. McDonnell (Wiley-Interscience, Chichester), 527

Grün, E. 2007, Solar System Dust, in Encyclopedia of the Solar System, ed. L.-A. McFadden, P. R. Weissman, \& T. V. Johnson 2nd edn. (San Diego: Academic Press-Elsevier Inc.), 621

Jackson, A. A., \& Zook, H. A. 1989, Nature, 337, 629

Klačka, J. 1994, Earth Moon Planets, 64, 125
Klačka, J. 2008a [arXiv:0807.2795]

Klačka, J. 2008b [arXiv:0807.2915]

Klačka, J., \& Saniga, M. 1993, Earth Moon Planets, 60, 23

Klačka, J., Petržala, J., Pástor, P., \& Kómar, L. 2009 [arXiv : 0904 . 0368]

Klačka, J., Kómar, L., Pástor, P., \& Petržala, J. 2008, A\&A, 489, 787

Klačka, J., \& Pástor, P. 2004 [arXiv:astro-ph/0411691]

Kuchner, M. J., \& Holman, M. J. 2003, ApJ, 588, 1110

Lafreniere, D., Jayawardhana, R., \& van Kerkwijk, M. H. 2008, ApJL, submitted [arXiv:0809.1424]

Leinert, Ch., \& Grün, E. 1990, Interplanetary dust, in Physics of the Inner Heliosphere I, ed. R. Schwen, \& E. Marsch (Berlin: Springer-Verlag), 207

Liou, J.-Ch., \& Zook, H. A. 1997, Icarus, 128, 354

Liou, J.-Ch., \& Zook, H. A. 1999, AJ, 118, 580

Liou, J.-Ch., Zook, H. A., \& Jackson, A. A. 1995, Icarus, 116, 186

Murray, C. D., \& Dermott, S. F. 1999, Solar System Dynamics (New York: Cambridge Univ. Press)

Neuhäuser, R., Guenther, E. W., \& Wuchterl, G., et al. 2005, A\&A, 435, L13

Parker, E. N. 1958, ApJ, 128, 664

Pástor, P., Klačka, J., \& Kómar, L. 2009, Cel. Mech. and Dynam. Astron., 103, 343

Poynting, J. M. 1903, MNRAS , 64, A 1

Reach, W. T., Franz, B. A., \& Welland, et al. 1995, Nature, 374, 521

Robertson, H. P. 1937, MNRAS, 97, 423

Seperuelo, E., Alencar, S. H. P., Batalha, C., \& Lopes, D. 2008, A\&A, 489, 349

Šidlichovský, M., \& Nesvorný, D. 1994, A\&A, 289, 972

Weidenschilling, S. J., \& Jackson, A. A. 1993, Icarus, 104, 244

Whipple, F. L. 1955, ApJ, 121, 750

Wyatt, S. P., \& Whipple, F. L. 1950, ApJ, 111, 134 